\title{
Study of Antenna Superstrates Using Metamaterials for Directivity Enhancement Based on Fabry-Perot Resonant Cavity
}

\author{
Haixia Liu, Shuo Lei, Xiaowei Shi, and Long Li \\ School of Electronic Engineering, Xidian University, Xian 710071, China \\ Correspondence should be addressed to Long Li; longliee@foxmail.com
}

Received 29 November 2012; Accepted 30 January 2013

Academic Editor: Duixian Liu

Copyright (C) 2013 Haixia Liu et al. This is an open access article distributed under the Creative Commons Attribution License, which permits unrestricted use, distribution, and reproduction in any medium, provided the original work is properly cited.

\begin{abstract}
Metamaterial superstrate is a significant method to obtain high directivity of one or a few antennas. In this paper, the characteristics of directivity enhancement using different metamaterial structures as antenna superstrates, such as electromagnetic bandgap (EBG) structures, frequency selective surface (FSS), and left-handed material (LHM), are unifiedly studied by applying the theory of Fabry-Perot (F-P) resonant cavity. Focusing on the analysis of reflection phase and magnitude of superstrates in presently proposed designs, the essential reason for high-directivity antenna with different superstrates can be revealed in terms of the F-P resonant theory. Furthermore, a new design of the optimum reflection coefficient of superstrates for the maximum antenna directivity is proposed and validated. The optimum location of the LHM superstrate which is based on a refractive lens model can be determined by the F-P resonant distance.
\end{abstract}

\section{Introduction}

Artificial electromagnetic materials, such as electromagnetic band gap (EBG) structures, frequency selective surface (FSS), and left-handed material (LHM), are broadly classified as metamaterials. They have attracted significant research interest in recent years due to their special electromagnetic properties, which are applicable to a wide range of electromagnetic devices. Array antennas had been widely used for applications requiring high directivity. However, array antennas require a complicated feeding network which makes difficulty in design and fabrication of array antennas.

Recent papers have proposed the use of metamaterials as superstrates for antennas to achieve gain enhancement [1-30]. Metamaterial superstrates are practically useful to enhance the directivity of different radiation sources, such as microstrip patch antennas $[1-8]$, waveguide antennas $[9,10]$, dipole antennas [11-14], slot antennas [3,15] and monopole antennas [16], and so forth. The Fabry-Perot (F-P) resonant cavity is generally formed by a metallic ground plane and a superstrate, which can be applied to antennas for directivity enhancement in an operational bandwidth. Conventionally, the profile of the F-P antenna is about half wavelength.
If using an artificial magnetic conductor (AMC) consisting of a periodic array of metallic patches on the ground plane, the F-P resonant cavity can reduce the height to $\lambda / 4$ [18]. A summary of recent researches on F-P antenna has been proposed [4], which shows that the analytic models of FP cavity, EBG defect model, transmission line model, leaky wave model, and refractive lens model are consistent when applied to analyze this type of resonator antenna. However, the analysis and design of the high-gain antenna with EBG as the cover are based on the defect modes [19, 20]. These modes involve in not only the superstrate but also its image through the ground plane of the antenna, which are not suitable for the composite structure of a single-layer FSS superstrate [22]. In addition, an LHM superstrate was also used to improve the radiation performance of an antenna, which was interpreted by the theory of near-zero refraction index materials $[23,26]$, but the theory is difficult to determine the optimum distance between the LHM superstrate and the antenna aperture.

In this paper, based on the theory of Fabry-Perot (F-P) resonant cavity, we further explain the unified mechanism of directivity enhancement using three different metamaterials as antennas' superstrates. By analyzing the reflection phase and magnitude of superstrates, we can design high-directivity 


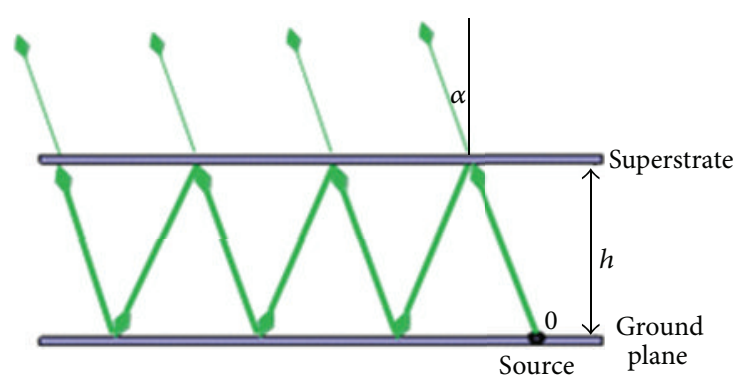

FIGURE 1: F-P resonant cavity composed of a ground plane and a superstrate.

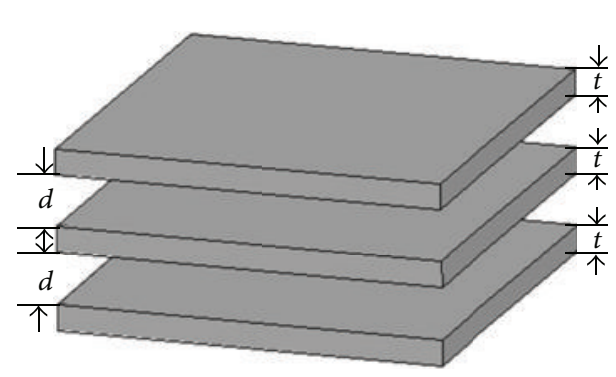

(a)

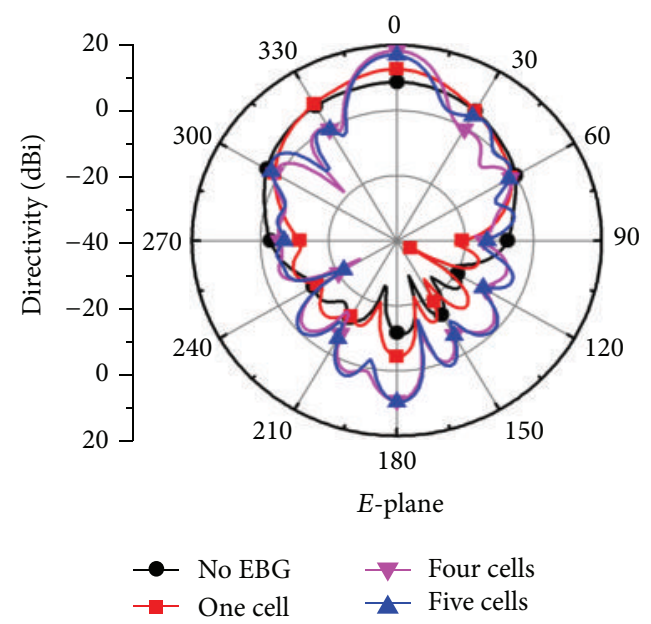

(c)

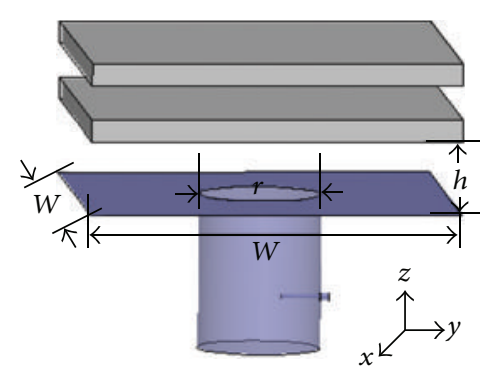

(b)

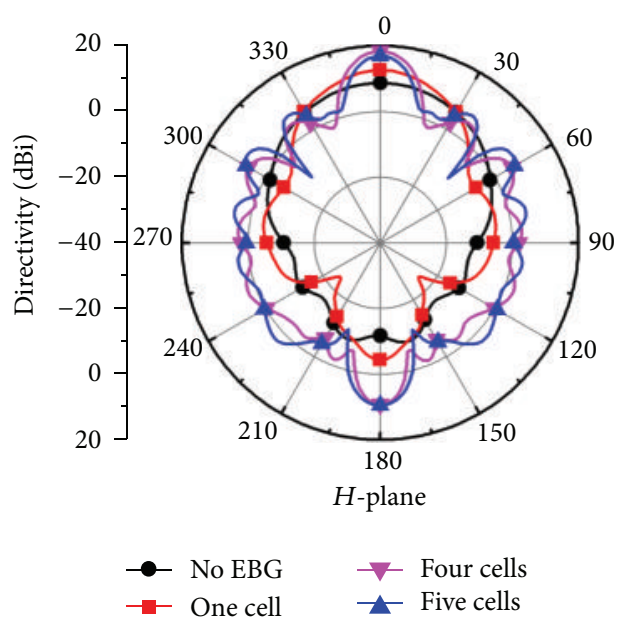

(d)

FIGURE 2: (a) Dielectric EBG structure, (b) circular waveguide antenna with EBG superstrate, (c) radiation patterns of $E$-plane with different EBG cells, (d) radiation patterns of $H$-plane with different EBG cells.

antennas with different types of superstrates according to F-P resonant theory. The organization of the paper is as follows. First, the theory of F-P resonat or antenna is briefly described, and the ideal optimum reflection magnitude of superstrate related to antenna maximum directivity is given. Second, taking EBG, FSS, and LHM superstrates proposed by previous references [24-26] for instance, we analyze the characteristics of different superstrates using full-wave simulation. An improved design method of the optimum reflection coefficient of the superstrate for the maximum antenna directivity is proposed in terms of the F-P cavity theory. Finally, the LHM superstrate is analyzed according to the theory of the Fabry-Perot resonant cavity and near-zero refractive theory of metamaterials.

\section{Theory of F-P Resonant Cavity}

A geometrical optics model has been applied to describe the theory of F-P resonator antenna [27, 28]. As shown in Figure 1, the conventional F-P cavity is composed of a ground plane and a superstrate placed with distance $h$. The ground plane has a complex reflection coefficient $e^{j \varphi_{1}}$, and the superstrate surface which is a partially reflection surface 


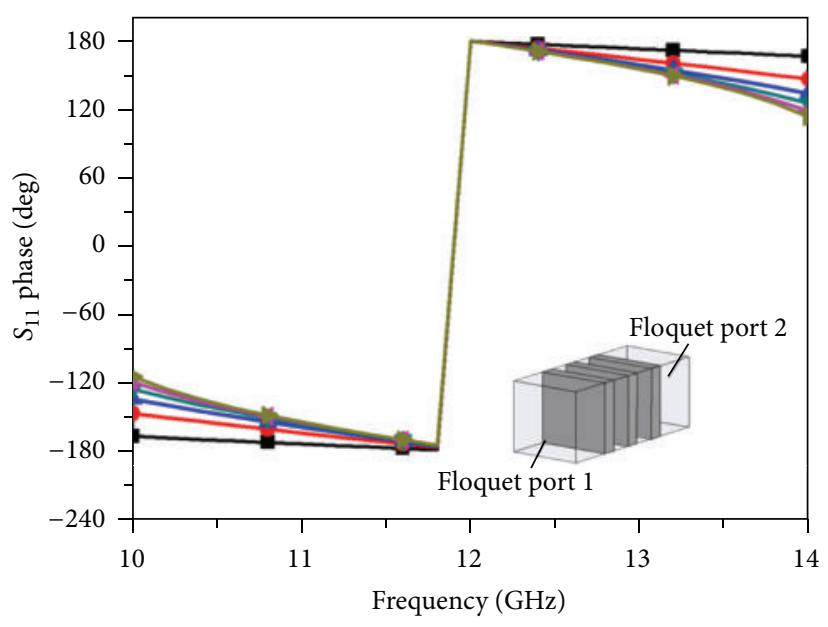

(a)

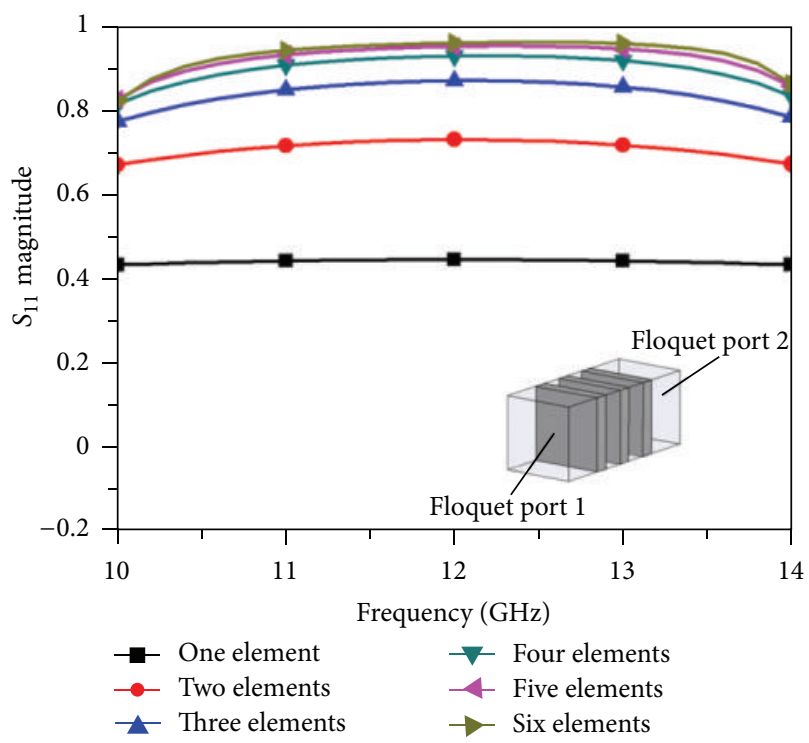

(b)

FIGURE 3: Simulated phase and magnitude of reflection coefficients $\left(S_{11}\right)$ of the dielectric EBG structure with different elements, (a) reflection phase (b) reflection magnitude.

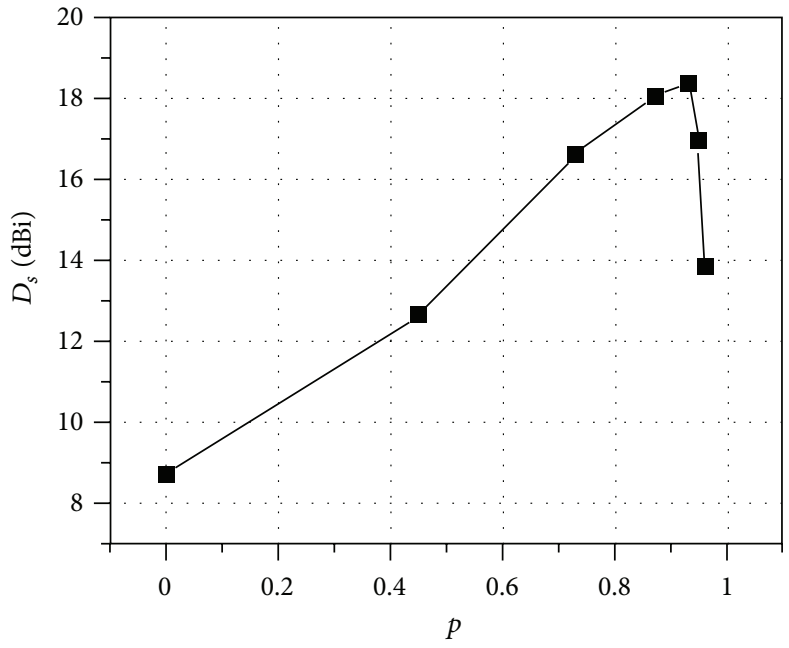

(a)

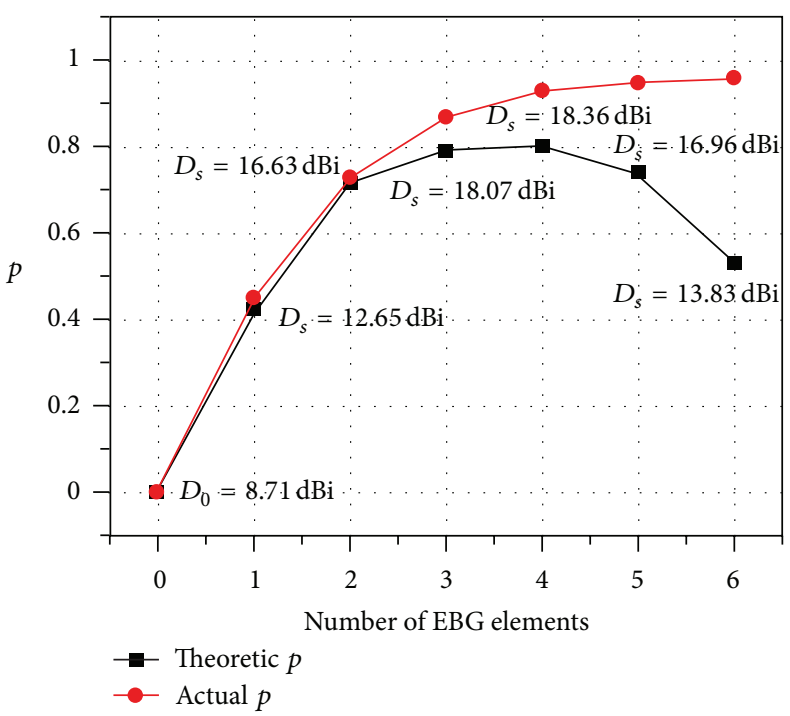

(b)

FIGURE 4: (a) Simulated directivity of the antenna with EBG superstrates versus different $p$, (b) comparison between the theoretic $p$ calculated by (10) and the actual $p$ realized by various EBG elements.

(PRS) has a complex reflection coefficient $p e^{j \varphi_{2}}$. Both of the two surfaces would be assumed homogeneous and ignored edge effects. According to the optics analysis model, the electromagnetic wave excited by a source will be bounced in the F-P cavity. Assuming no transmission loss, the electric field intensity in the far zone consists of the vector sum of these partial rays, and for an infinite screen and sheet we may write $[27,28]$

$$
E=\sum_{n=0}^{\infty} f(\alpha) E_{0} p^{n} \sqrt{1-p^{2}} e^{j \Theta_{n}},
$$

where $f(\alpha)$ is the antenna pattern function in $\alpha$ direction. The phase angle $\Theta_{n}$ is composed of the phase variations during reflections from the completely reflecting screen and partially reflecting sheet, and also of the path differences of the partial rays as follows:

$$
\Theta_{n}=n \Phi=n\left(-\frac{4 \pi}{\lambda} l \cos \alpha+\varphi_{1}+\varphi_{2}\right),
$$

where $\lambda$ is the operating wavelength. The power pattern is therefore

$$
S=\frac{1-p^{2}}{1+p^{2}-2 p \cos \left(\varphi_{1}+\varphi_{2}-(4 \pi / \lambda) h \cos \alpha\right)} f^{2}(\alpha) .
$$




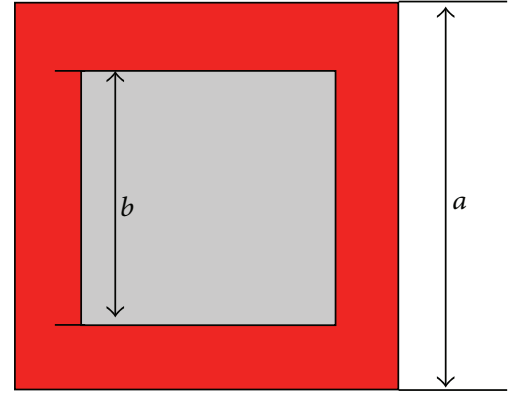

(a)

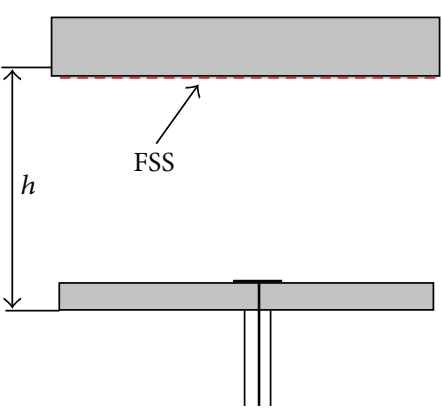

(b)

FIGURE 5: (a) Unit cell of the FSS and (b) patch antenna with FSS superstrate.

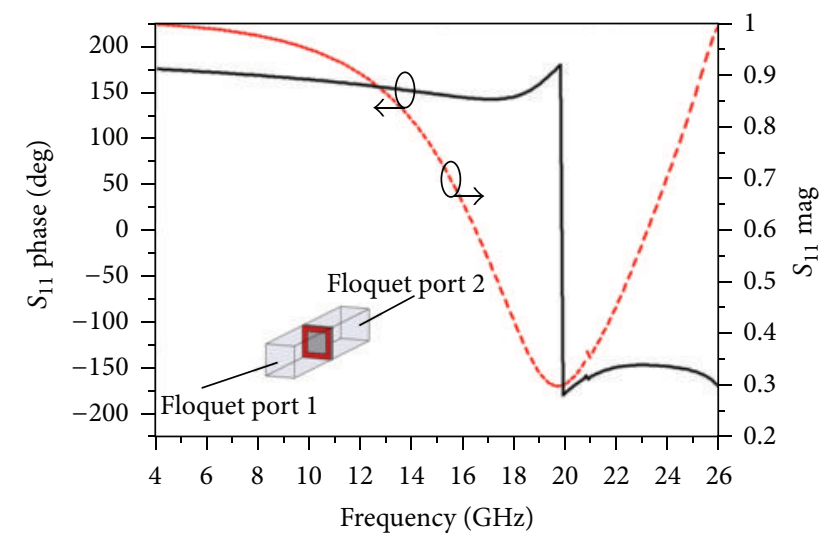

FIGURE 6: Reflection coefficient $\left(S_{11}\right)$ phase and magnitude of the FSS.

Consider the forward direction, that is, $\alpha=0^{\circ}$, then

$$
S=\frac{1-p^{2}}{1+p^{2}-2 p \cos \left(\varphi_{1}+\varphi_{2}-(4 \pi / \lambda) h\right)} f^{2}(0)
$$

when

$$
\cos \left(\varphi_{1}+\varphi_{2}-\frac{4 \pi}{\lambda} h\right)=1
$$

which is corresponding to the resonant condition. The resonant distance can be written as

$$
h=\frac{\varphi_{1}+\varphi_{2}}{\pi} \frac{\lambda}{4}+N \frac{\lambda}{2}, \quad N=0,1,2, \ldots
$$

Therefore, maximum power in the direction of $\alpha=0^{\circ}$ can be obtained as follows:

$$
S_{\max }=\frac{1+p}{1-p} f^{2}(0)=\frac{1+p}{1-p} S_{0},
$$

where $S_{0}$ is corresponding to the forward power from the source without superstrate.

It is shown that the resonant cavity height $h$ can be determined by the reflection phase $\varphi_{1}, \varphi_{2}$, and the operating frequency $\lambda$. In the F-P cavity antenna system, $e^{j \varphi_{1}}$ is the complex reflection coefficient of the ground plane of an antenna, and $p e^{j \varphi_{2}}$ is the complex reflection coefficient of the superstrate which may be composed of EBG, FSS, or LHM structures. Conventionally, the ground plane is a metallic conductor with reflection phase close to $\pi\left(\varphi_{1} \approx \pi\right)$. Therefore, the resonance condition of (6) can be reduced as follows:

$$
h=\left(1+\frac{\varphi_{2}}{\pi}\right) \frac{\lambda}{4}
$$

when $N=0$. If the reflection phase $\varphi_{2}$ of the superstrate and the operating frequency $\lambda$ are given, the resonant distance $h$ will be determined. At the resonant distance $h$, the antenna system with a metamaterial superstrate can achieve a high directivity, as well as high gain.

Furthermore, we assume that an original planar antenna without superstrate has a directivity of $D_{0}$ at operating frequency $f_{0}$, and the F-P cavity antenna which consists of the same planar antenna and a superstrate has directivity of $D_{s}$ at the same frequency. The amount of improvement of the directivity can be approximately written as

$$
\Delta D(\mathrm{~dB})=D_{s}(\mathrm{~dB})-D_{0}(\mathrm{~dB})=10 \log _{10} \frac{1+p}{1-p} .
$$

Therefore, the magnitude of reflection coefficient $p$ of the superstrate can be obtained from

$$
p=\frac{10^{\Delta D(\mathrm{~dB}) / 10}-1}{10^{\Delta D(\mathrm{~dB}) / 10}+1} .
$$

For an aperture antenna, the ideal maximum directivity $D_{\max }$ can be estimated by

$$
D_{\max }(\mathrm{dB})=10 \log _{10} \frac{4 \pi A_{\mathrm{em}}}{\lambda_{0}^{2}},
$$

where $A_{\mathrm{em}}$ is the maximum effective aperture of the antenna. For the F-P cavity antenna, it is reasonable to assume that $A_{\mathrm{em}}$ is the maximum physical area of the superstrate. Therefore, the ideal optimum reflection magnitude of the superstrate is related to its maximum directivity by

$$
p_{\text {opt }}=\frac{10^{\left[D_{\max }(\mathrm{dB})-D_{0}(\mathrm{~dB})\right] / 10}-1}{10^{\left[D_{\max }(\mathrm{dB})-D_{0}(\mathrm{~dB})\right] / 10}+1} .
$$




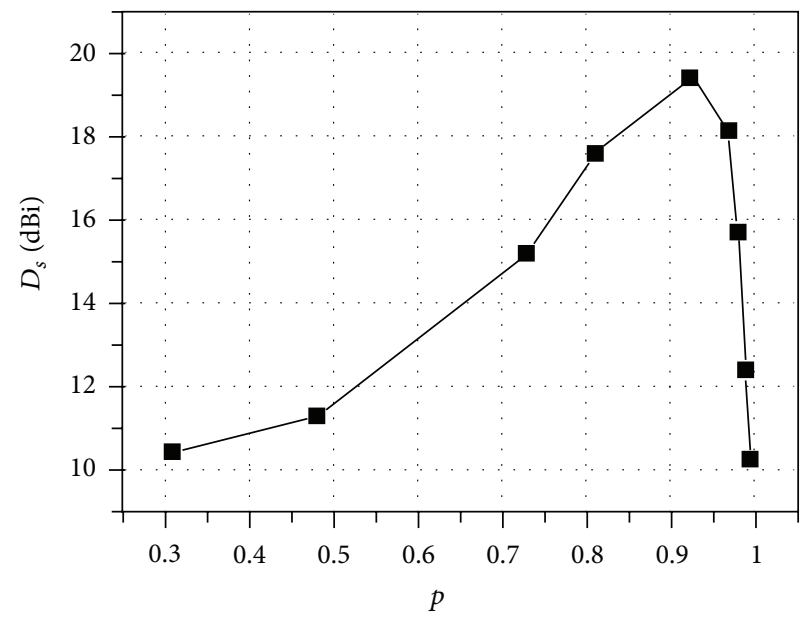

(a)

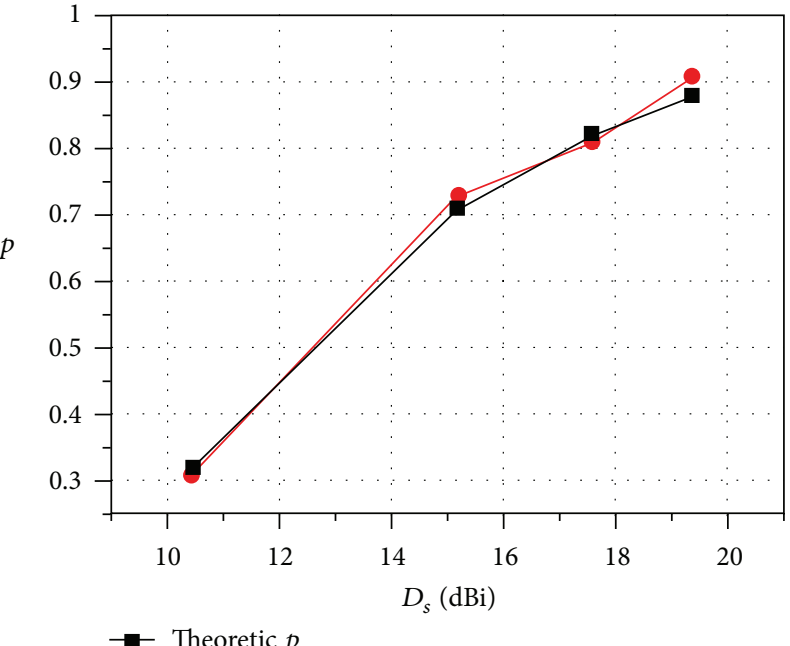

Actual $p$

FIGURE 7: (a) Directivity of the patch antenna with FSS superstrates versus different $p$, (b) comparison of the theoretic and the actual $p$ corresponding to the antenna directivity enhancement.

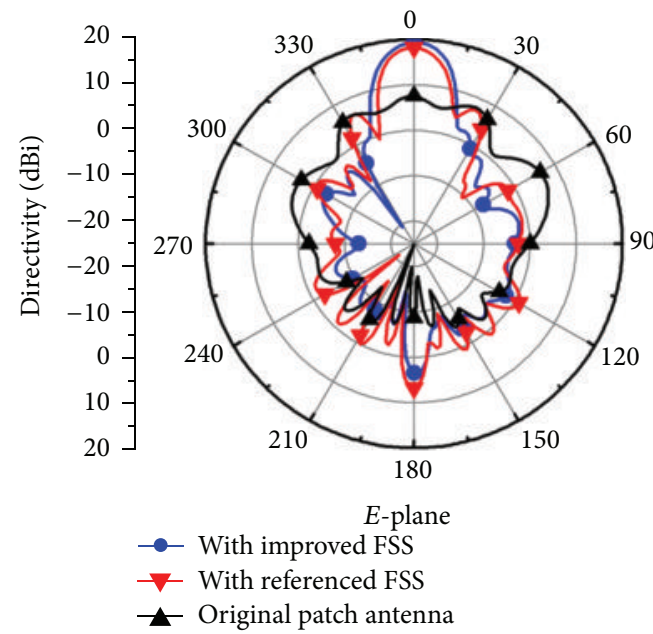

(a)

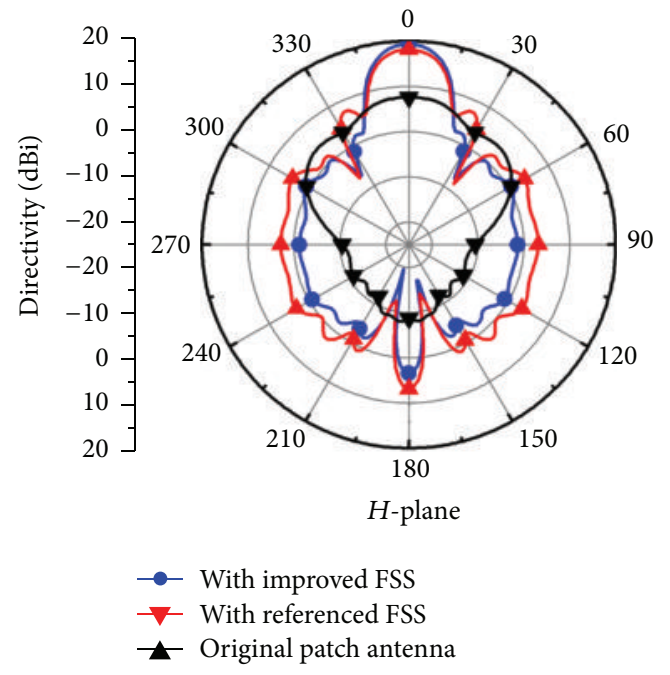

(b)

FIGURE 8: Comparison of directivities patterns of different FSS superstrates, (a) E-plane and (b) $H$-plane.
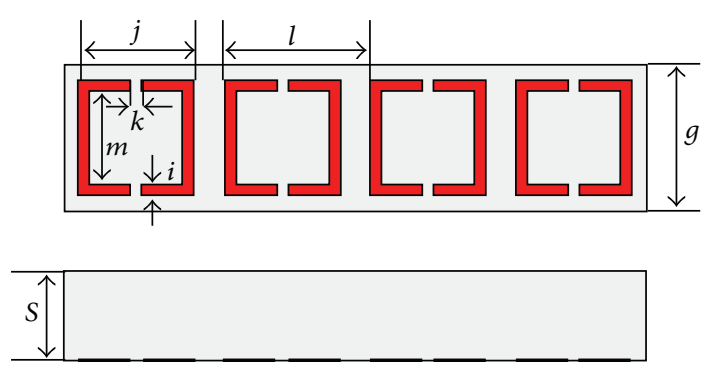

FIGURE 9: Geometry of LHM structure.

This assumes that there are no conduction-dielectric losses and the antenna is matched to the load. It provides a primary principle guiding the design to achieve the maximum directivity of the F-P cavity antenna with supstrates based on (12). Numerical experiments in the next section show that when the reflection coefficient $p$ of a real superstrate is less than $p_{\text {opt }}$, the larger $p$ of the superstrate is chosen, the higher directivity of the F-P cavity antenna can be obtained. However, when the designed $p$ is more than $p_{\text {opt }}$, the directivity of the F-P cavity antenna will decrease with $p$ further increased.

\section{Analysis of Three Kinds of Different Metamaterial Superstrates}

Taking into account three kinds of different metamaterial superstrates proposed by [24-26], respectively, we give a 


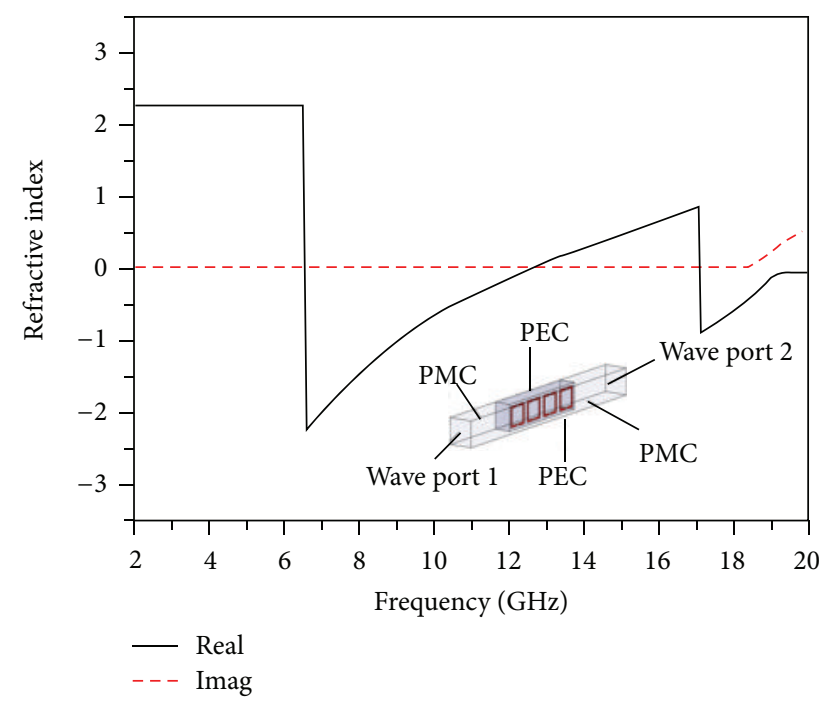

FIGURE 10: Effective refractive index of the LHM structure.

detailed analysis of the characteristics of different superstrates and their relation to the directivity enhancement of antennas by using the theory of F-P cavity.

3.1. Dielectric EBG Superstrate. A typical design example using one-dimension periodicity dielectric EBG superstrate proposed by [24] is shown in Figure 2(a). The dimension of the dielectric EBG superstrate is rewritten here $t=3.84 \mathrm{~mm}$, $d=6.25 \mathrm{~mm}$, the cross section is $60 \mathrm{~mm} \times 60 \mathrm{~mm}$, and the relative dielectric permittivity is 2.65 . When the EBG superstrate has a distance $13.2 \mathrm{~mm}$ to the antenna ground plane (Figure 2(b)), the circular waveguide aperture antenna, whose dimension is $w=60 \mathrm{~mm}$ and $r=20 \mathrm{~mm}$, can achieve a high gain at $12 \mathrm{GHz}$. Moreover, the paper pointed out that the antenna gain would increase with the increase of EBG elements when the number of EBG elements is less than four [24]. Meanwhile it means that the superstrate with more EBG elements is not always better. The comparisons of the radiation patterns of $E$-plane ( $y z$ plane) and $H$-plane ( $x z$ plane) of the circular waveguide antenna with EBG superstrates composed of different EBG cells are given in Figures 2(c) and 2(d).

Here, we will provide an insight into the above phenomenon in terms of the reflection phase and magnitude of the EBG superstrate based on the theory of F-P resonant cavity. A full-wave EM simulation was performed by using finite-element analysis based on HFSS [30]. The periodic boundary conditions (PBCs) and Floquet ports are used to simulate the infinite periodic model of the EBG superstrate. Figure 3 shows that the reflection phase $\varphi_{2}$ and the reflection magnitude $p$ of different number of EBG elements. It can be seen from Figure 3(a) that the EBG superstrate with different number of elements has almost the same reflection phase $\varphi_{2}$ at the working frequency $12 \mathrm{GHz}$, that is, $\varphi_{2} \approx \pi$. Inserting $\varphi_{2}$ and $\lambda$ at $12 \mathrm{GHz}$ into (8), we obtain the resonant height of the EBG superstrate should be $h=12.5 \mathrm{~mm}$, which is in agreement with the distance given by [24]. It is revealed that the antenna with EBG superstrate system satisfied the resonance condition of F-P cavity. On the other hand, Figure $3(\mathrm{~b})$ shows that the magnitude $p$ of reflection coefficient $\left(S_{11}\right)$ increases with the increase of the EBG elements.

The directivities of the antenna with the EBG superstrate versus different actual $p$ are simulated and shown in Figure 4(a). Note that the original circular waveguide antenna without superstrate has a directivity of $D_{0}=$ $8.71 \mathrm{dBi}$, in which case corresponds to $p=0$ (without superstrate). When the superstrate has four EBG elements, in which case $p$ is about 0.93 , the directivity is $D_{s}=18.36 \mathrm{dBi}$. Figure 4(b) shows that the comparison between the theoretic $p$ calculated by (10) and the actual $p$ realized by various EBG elements. It can be seen that the theoretical prediction $p$ is in accordance with the result of actual $p$ when the superstrates are composed of one or two EBG elements, which indicates the suitable condition of F-P cavity theory for EBG superstrates. Nevertheless, the ideal maximum directivity $D_{\max }$ and $p_{\text {opt }}$ of the circular waveguide aperture antenna with the EBG superstrate can be still predicted according to (11) and (12), respectively. In this case, $D_{\max }$ is $18.6 \mathrm{dBi}$ at $12 \mathrm{GHz}$ and $p_{\text {opt }}$ is 0.82 . It can be seen from Figure 4(a) that the actual maximum directivity $D_{s}$ is $18.36 \mathrm{dBi}$, which is very close to the ideal maximum directivity $D_{\max }$. When the number of elements of the EBG superstrate is increased continuously, the corresponding $p$ is greater than the $p_{\mathrm{opt}}$ so that the directivity enhancement will decrease. It can be concluded that when the reflection coefficient $p$ of the designed superstrate is less than $p_{\text {opt }}$, the larger $p$ of the superstrate is chosen, the higher directivity of the F-P cavity antenna can be obtained. However, when the actual $p$ is more than $p_{\text {opt }}$, the directivity of the F-P cavity antenna will decrease with $p$ further increased. Therefore, by analyzing the reflection phase and magnitude of the EBG superstrate, it is revealed that the mechanism of gain enhancement using EBG dielectric structure as the antenna superstrate is intrinsically based on the theory of F-P resonant cavity. It is worth pointing out that the directivities mentioned above are gotten when the EBG superstrates are assumed to be lossless. If the EBG superstrates were lossy with $\tan \delta=0.002$, the computed $D_{\max }$ would be $18.15 \mathrm{dBi}$ at $12 \mathrm{GHz}$. So it is shown that the loss of the superstrate has a small influence on the directivity enhancement.

3.2. FSS Superstrate. FSS structures can also be used as antenna superstrate to enhance antenna directivity. A FSS structure of square patches proposed by [25] is shown in Figure 5(a), and the distance between the FSS superstrate and the microstrip antenna ground plane is about $15 \mathrm{~mm}$. In this case, the measured maximum gain of the patch antenna with FSS superstrate is $16.5 \mathrm{dBi}$ at $8.7 \mathrm{GHz}$. The FSS is composed of an Arlon Diclad dielectric slab having relative permittivity of 2.5, tangent loss of 0.0022 , with thickness of $1.59 \mathrm{~mm}$. The periodicity of the square patch array is $a=10 \mathrm{~mm}$ and $b=6.5 \mathrm{~mm}$. We analyzed the reflection phase $\varphi_{2}$ and the reflection magnitude $p$ of the FSS superstrate using infinite periodic model simulation, as shown in Figure 6. It can 


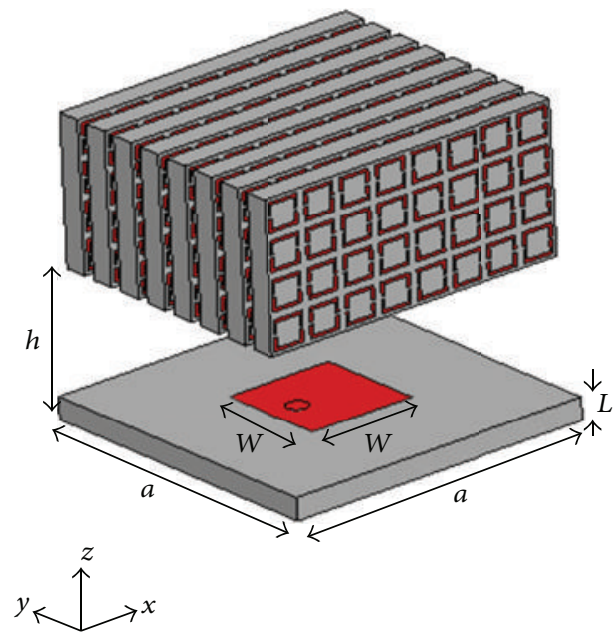

(a)

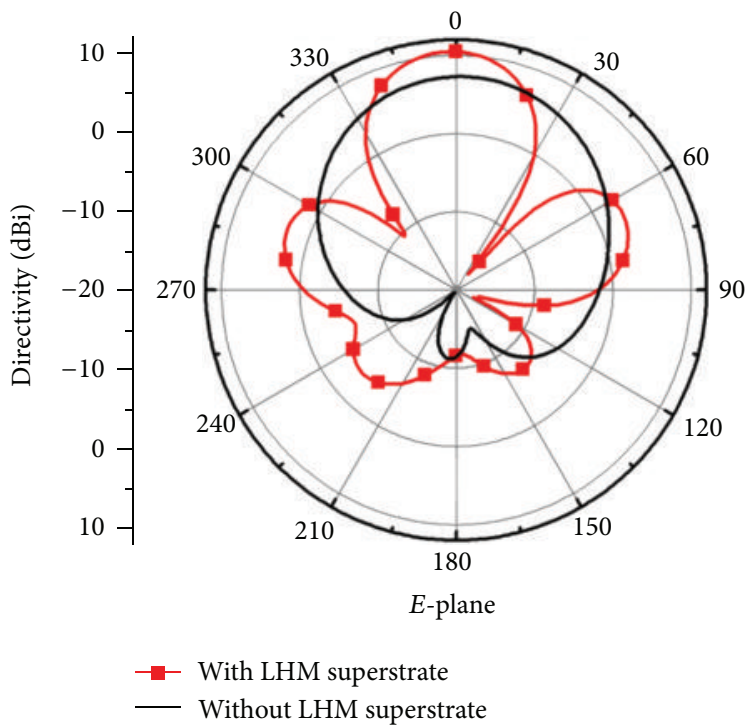

(c)

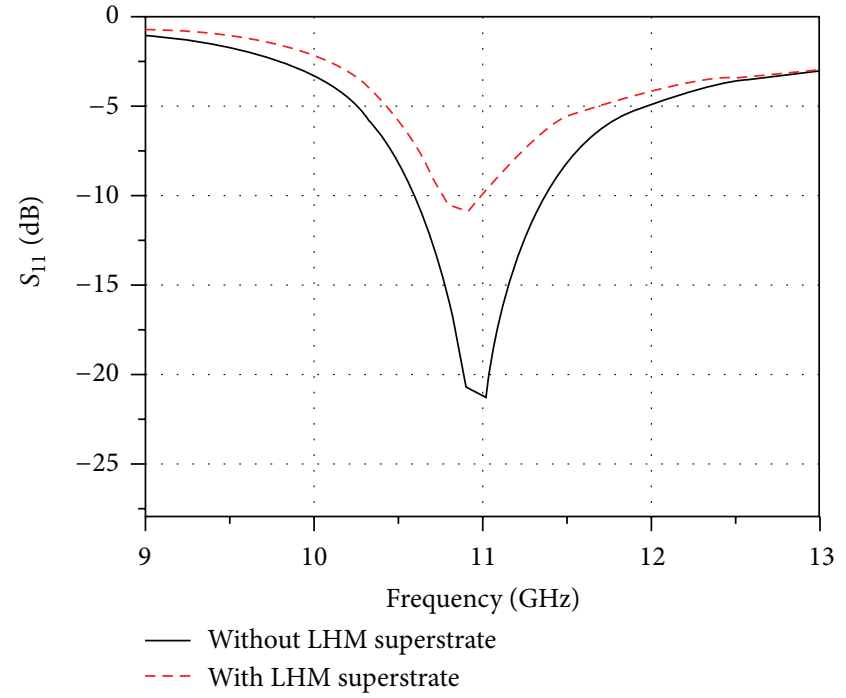

(b)

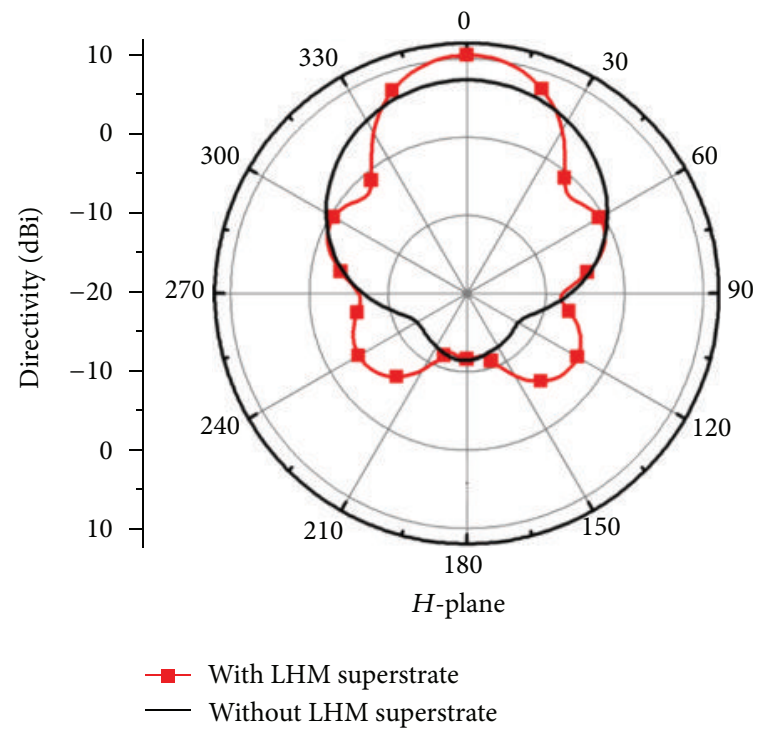

(d)

Figure 11: (a) Patch antenna with LHM superstrate, (b) $S_{11}$ characteristics, (c) comparison of directivity E-plane patterns ( $x z$ plane) with and without the LHM superstrate, (d) comparison of directivity $H$-plane patterns ( $y z$ plane) with and without the LHM superstrate.

be seen that the reflection phase $\varphi_{2}$ of the FSS superstrate is $166^{\circ}$ at $8.7 \mathrm{GHz}$. The FSS superstrate is placed above a microstrip antenna whose substrate dielectric is also Arlon Diclad and cross section area is $150 \mathrm{~mm} \times 150 \mathrm{~mm}$, as shown in Figure 5(b). Similarly, inserting $\varphi_{2}$ and $\lambda$ at $8.7 \mathrm{GHz}$ in (8), we can determine that the resonant distance $h$ should be $16.5 \mathrm{~mm}$, which is also in good agreement with the distance given in [25]. Thus it can be concluded that the design of FSS superstrate for high-directivity antenna is also dependent on the theory of F-P resonant cavity. Besides, it is found that the operating frequency band of the FSS superstrate used for the directivity enhancement is in its stopband but not its passband.

Analyzing the reflection magnitude of the FSS superstrate, we can see that the actual $p$ is 0.964 when $\varphi_{2}=166^{\circ}$.
Putting the FSS structure at the resonant height, the patch antenna can get a high directivity of $18.1 \mathrm{dBi}$ by simulation. According to (11) and (12), the ideal maximum directivity $D_{\max }$ and optimum reflection magnitude $p_{\text {opt }}$ should be $23.7 \mathrm{dBi}$ and 0.95 , respectively.

It can be predicted from F-P cavity theory that the original FSS design is not optimal, because its reflection magnitude $p$ is more than $p_{\text {opt }}$. By adjusting the patch width $b$ of the FSS structure, we calculated the corresponding relationship of reflection coefficient magnitude $p$ with the antenna directivity, as shown in Figure 7(a). It is revealed that when $p=0.924$, the actual maximum directivity of $19.4 \mathrm{dBi}$ can be achieved, which is higher than that of the proposed design [25] by $1.3 \mathrm{~dB}$. The dimension of the modified FSS superstrate is $a=$ $10 \mathrm{~mm}$ and $b=6.9 \mathrm{~mm}$. The comparisons of the radiated 
patterns and directivities of the original patch antenna, the patch antenna with the FSS superstrate proposed by [25], and the patch antenna with our improved FSS superstrate, are shown in Figure 8. The comparison of the theoretic $p$ calculated by (10) and the actual $p$ of FSS simulated by HFSS corresponding to different antenna directivity is shown in Figure 7(b). The good agreement of the two curves furthermore verifies the correction and effectiveness of the optimum reflection magnitude design based on F-P cavity theory.

3.3. LHM Superstrate. Generally, the analysis of LHM superstrate for directivity enhancement of an antenna is based on the effective near-zero refractive index of metamaterials. However, it is still difficult to determine the optimum distance between LHM superstrate and antenna aperture. In this section, we give an insight into the mechanism of the LHM superstrate by combining the near-zero refraction theory with Fabry-Perot resonant cavity theory.

The LHM structure proposed by [26] is shown in Figure 9, whose parameters are as follows:

$$
\begin{gathered}
g=2.5 \mathrm{~mm}, \quad i=0.2 \mathrm{~mm}, \quad j=2 \mathrm{~mm}, \quad k=0.2 \mathrm{~mm}, \\
l=2.5 \mathrm{~mm}, \quad s=0.75 \mathrm{~mm}, \quad \varepsilon_{r}=4.4 .
\end{gathered}
$$

It has been pointed out that the antenna gain would increase when the LHM superstrate operates at $11 \mathrm{GHz}$. By analyzing the reflection and transmission characteristics of the LHM structure, we extracted the effective refractive index [31], as shown in Figure 10. It can be noted that the refractive index is close to zero around $11 \mathrm{GHz}$, and the electromagnetic wave can be concentrated around the normal of the LHM slab. The proposed LHM structure was used as the superstrate for a patch antenna, as illustrated in Figure 11(a). The original patch antenna has the following geometry parameter: $a=$ $20 \mathrm{~mm}, w=7 \mathrm{~mm}, L=1.5 \mathrm{~mm}$, and $\varepsilon_{r}=2.94$, whose directivity is $7.3 \mathrm{dBi}$ at the working frequency of $11 \mathrm{GHz}$. The reflected coefficient $S_{11}$ and radiated patterns are simulated and shown in Figures 11(b), 11(c), and 11(d). By adjusting the position of the LHM superstrate, we can determine the optimum distance, that is, $h=12.4 \mathrm{~mm}$, to achieve the maximum directivity of the antenna, as shown in Figure 12. The $S_{11}$ and radiated directivity patterns of the patch antenna with the LHM superstrate $(h=12.4 \mathrm{~mm})$ are also shown in Figures 11(b) and 11(c) for comparison.

From another point of view, we consider the antenna system with LHM superstrate as the F-P resonant cavity antenna approximately. We first analyze the reflection phase $\varphi_{2}$ and the reflection magnitude $p$ of the LHM superstrate, as shown in Figure 13. Note that the reflection phase $\varphi_{2}$ is $148.5^{\circ}$ at the frequency of $11 \mathrm{GHz}$. Here, inserting the $\varphi_{2}$ and $\lambda$ at $11 \mathrm{GHz}$ into (8), we can obtain the resonant distance $h=12.4 \mathrm{~mm}$ between the LHM superstrate and the patch antenna aperture, which is just corresponding to the optimum distance determined by numerical simulations shown in Figure 12.

It is revealed that the maximum directivity of the antenna system can be achieved by setting the LHM superstrate at

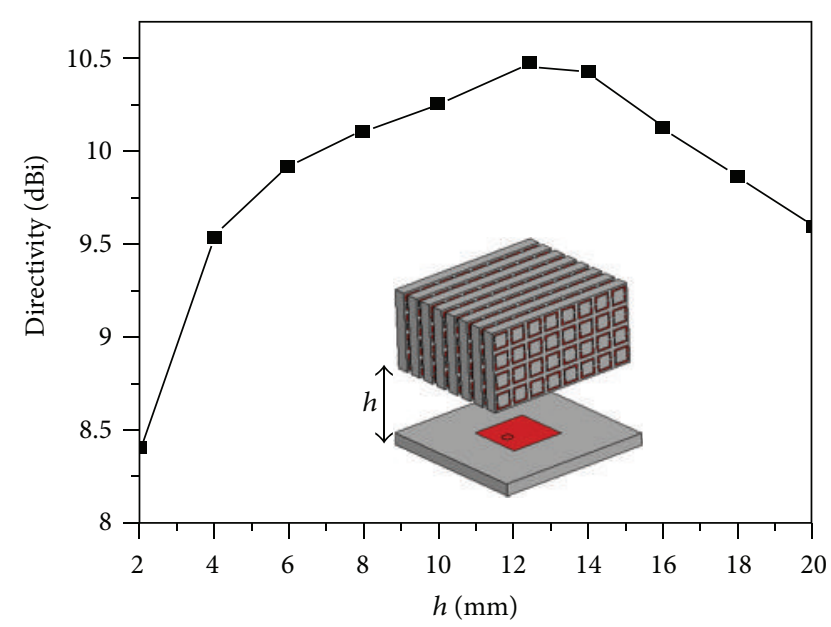

FIGURE 12: Directivities of the patch antenna with LHM superstrate located at different distance.

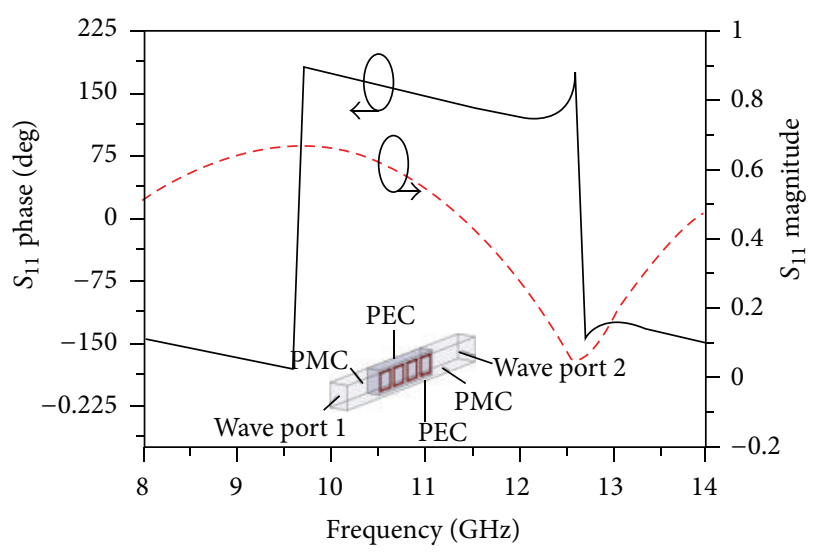

FIGURE 13: Reflection phase and magnitude of the LHM superstrate.

the resonant height of the F-P resonant cavity. When the LHM superstrate is placed at another position, the directivity enhancement would be less than that at the resonant position, which is only contributed from the near-zero refraction index response. Therefore, it is shown that we can obtain an optimum design of the LHM superstrate by combining the F$\mathrm{P}$ resonant cavity theory with the near-zero refractive index of metamaterials. In addition, it is found that the directivity enhancement of the LHM superstrate is weak compared with the EBG and FSS superstrates. The reason for this is that the LHM superstrate has a small reflection coefficient magnitude at the working frequency, that is, $p=0.55$, which cannot achieve a high directivity according to the theory of F-P resonant cavity.

\section{Conclusion}

In this paper, the mechanism of directivity enhancement using different metamaterial structures as antenna superstrates was studied by unifiedly applying the theory of Fabry-Perot (F-P) resonant cavity. An improved design of 
superstrates for the maximum antenna directivity enhancement is proposed and validated. Based on the analysis of the reflection magnitude and phase of superstrates, three kinds of metamaterial superstrates, such as EBG structure, FSS, and LHM, are proved to satisfy the theory of Fabry-Perot resonant cavity. It has been shown that if the reflection magnitude $p$ of the EBG superstrate is less than the ideal optimum reflection magnitude $p_{\text {opt }}$, the increase of EBG elements brings about the enhancement of the antenna directivity. While it is more than the optimum reflection coefficient, the antenna directivity would decrease with the increase of EBG elements. For the FSS superstrate, it also has an optimum reflection coefficient that makes the antenna obtain the maximum directivity. According to the F-P cavity theory, the operating frequency of the FSS superstrate used for the directivity enhancement is in its stopband but not the passband. The LHM used to improve antenna directivity is based on both the F-P resonant cavity and the near-zero refractive index of metamaterials. The optimum distance of the LHM superstrate for maximum directivity is also determined by the F-P resonant distance.

\section{Acknowledgments}

This work is supported partly by the Program for New Century Excellent Talents in University of China, and partly supported by the National Natural Science Foundation of China under Contract no. 61072017 and no. 61072021, National Key Laboratory Foundation, the Fundamental Research Funds for the Central Universities, and Foundation for the Returned Overseas Chinese Scholars, State Education Ministry and Shaanxi Province.

\section{References}

[1] A. Foroozesh and L. Shafai, "Investigation into the effects of the reflection phase characteristics of highly-reflective superstrates on resonant cavity antennas," IEEE Transactions on Antennas and Propagation, vol. 58, no. 10, pp. 3392-3396, 2010.

[2] A. R. Weily, L. Horvath, K. P. Esselle, B. C. Sanders, and T. S. Bird, "A planar resonator antenna based on a woodpile EBG material," IEEE Transactions on Antennas and Propagation, vol. 53, no. 1, pp. 216-223, 2005.

[3] Z. G. Liu, "Fabry-Perot resonator antenna," Journal of Infrared, Millimeter and Terahertz Waves, vol. 31, pp. 391-403, 2010.

[4] M. Thèvenot, C. Cheype, A. Reineix, and B. Jecko, "Directive photonic-bandgap antennas," IEEE Transactions on Microwave Theory and Techniques, vol. 47, no. 11, pp. 2115-2122, 1999.

[5] R. Mittra, Y. Li, and K. Yoo, "A comparative study of directivity enhancement of microstrip patch antennas with using three different superstrates," Microwave and Optical Technology Letters, vol. 52, no. 2, pp. 327-331, 2010.

[6] N. Guérin, S. Enoch, G. Tayeb, P. Sabouroux, P. Vincent, and H. Legay, "A metallic Fabry-Perot directive antenna," IEEE Transactions on Antennas and Propagation, vol. 54, no. 1, pp. 220-224, 2006.

[7] A. Foroozesh and L. Shafai, "Investigation into the effects of the patch-type FSS superstrate on the high-gain cavity resonance antenna design," IEEE Transactions on Antennas and Propagation, vol. 58, no. 2, pp. 258-270, 2010.
[8] H. Y. Yang and N. G. Alexopoulos, "Gain enhancement methods for printed circuit antennas through multiple superstrates," IEEE Transactions on Antennas and Propagation, vol. 35, no. 7, pp. 860-863, 1987.

[9] Y. Li and K. P. Esselle, "Small EBG resonator high-gain antenna using in-phase highly-reflecting surface," Electronics Letters, vol. 45, no. 21, pp. 1058-1060, 2009.

[10] A. P. Feresidis and J. C. Vardaxoglou, "High gain planar antenna using optimised partially reflective surfaces," IEE Proceedings, vol. 148, no. 6, pp. 345-350, 2001.

[11] E. Rodes, M. Diblanc, E. Arnaud, T. Monédière, and B. Jecko, "Dual-band EBG resonator antenna using a single-layer FSS," IEEE Antennas and Wireless Propagation Letters, vol. 6, pp. 368371, 2007.

[12] S. Wang, A. P. Feresidis, G. Goussetis, and J. C. Vardaxoglou, "High-gain subwavelength resonant cavity antennas based on metamaterial ground planes," IEE Proceedings, vol. 153, no. 1, pp. $1-6,2006$.

[13] L. Zhou, H. Li, Y. Qin, Z. Wei, and C. T. Chan, "Directive emissions from subwavelength metamaterial-based cavities," Applied Physics Letters, vol. 86, no. 10, Article ID 101101, pp. 1-3, 2005.

[14] E. Sáenz, R. Gonzalo, I. Ederra, J. C. Vardaxoglou, and P. de Maagt, "Resonant meta-surface superstrate for single and multifrequency dipole antenna arrays," IEEE Transactions on Antennas and Propagation, vol. 56, no. 4, pp. 951-960, 2008.

[15] A. R. Weily, K. P. Esselle, T. S. Bird, and B. C. Sanders, "High gain circularly polarised 1-D EBG resonator antenna," Electronics Letters, vol. 42, no. 18, pp. 1012-1013, 2006.

[16] S. Enoch, G. Tayeb, P. Sabouroux, and P. Vincont, "A metamaterial for directive emission," Physical Review Letters, vol. 89, no. 21, Article ID 213902, 2002.

[17] M. Qiu and S. He, "High-directivity patch antenna with both photonic bandgap substrate and photonic bandgap cover," Microwave and Optical Technology Letters, vol. 30, no. 1, pp. 4144, 2001.

[18] A. P. Feresidis, G. Goussetis, S. Wang, and J. C. Vardaxoglou, "Artificial magnetic conductor surfaces and their application to low-profile high-gain planar antennas," IEEE Transactions on Antennas and Propagation, vol. 53, no. 1, pp. 209-215, 2005.

[19] C. Cheype, C. Serier, M. Thèvenot, T. Monédière, A. Reineix, and B. Jecko, "An electromagnetic bandgap resonator antenna," IEEE Transactions on Antennas and Propagation, vol. 50, no. 9, pp. 1285-1290, 2002.

[20] Y. J. Lee, J. Yeo, R. Mittra, and W. S. Park, “Thin Frequency Selective Surface (FSS) superstrate with different periodicities for dual-band directivity enhancement," in Proceedings of the IEEE International Workshop on Antenna Technology: Small Antennas and Novel Metamaterials (IWAT '05), pp. 375-378, March 2005.

[21] A. Pirhadi, F. Keshmiri, M. Hakkak, and M. Tayarani, "Analysis and design of dual band high directivity EBG resonator antenna using square loop FSS AS superstrate layer," Progress in Electromagnetics Research, vol. 70, pp. 1-20, 2007.

[22] K. Yoo, R. Mittra, and N. Farahat, "A novel technique for enhancing the directivity of microstrip patch antennas using an EBG superstrate," in Proceedings of the IEEE Antennas and Propagation Society International Symposium, pp. 1-4, July 2008.

[23] B. Li, B. Wu, and C. H. Liang, "Study on high gain circular waveguide array antenna with metamaterial structure," Progress in Electromagnetics Research, vol. 60, pp. 207-219, 2006. 
[24] B. Li, X. J. Dang, and C. H. Liang, "High gain circular waveguide antenna using 1-D electromagnetic band-gap structure," Chinese Journal of Radio Science, vol. 20, no. 6, pp. 879-884, 2006.

[25] A. Foroozesh and L. Shafai, "On the design of high-gain resonant cavity antennas using different highly-reflective frequency selective surfaces as the superstrates," in Proceedings of the IEEE Antennas and Propagation Society International Symposium, July 2010.

[26] Y. Zhao, G. Wan, H. Zhao, and W. Zheng, "Effects of superstrate with improved SSRRs on the radiation of microstrip antenna," in Proceedings of the 3rd IEEE International Symposium on Microwave, Antenna, Propagation and EMC Technologies for Wireless Communications (MAPE '09), pp. 51-54, October 2009.

[27] G. von Trentini, "Partially reflecting sheet arrays," IRE Transactions on Antennas and Propagation, vol. 4, pp. 666-671, 1956.

[28] H. Boutayeb, K. Mahdjoubi, A. C. Tarot, and T. A. Denidni, "Directivity of an antenna embedded inside a Fabry-Perot cavity: analysis and design," Microwave and Optical Technology Letters, vol. 48, no. 1, pp. 12-17, 2006.

[29] D. Kim and J. I. Choi, "Analysis of a high-gain Fabry-Pérot cavity antenna with an fss superstrate: effective medium approach," Progress in Electromagnetics Research Letters, vol. 7, pp. 59-68, 2009.

[30] High Frequency Structure Simulator, Ansys Corporation.

[31] D. R. Smith, D. C. Vier, T. Koschny, and C. M. Soukoulis, "Electromagnetic parameter retrieval from inhomogeneous metamaterials," Physical Review E, vol. 71, no. 3, Article ID 036617, pp. 1-11, 2005. 

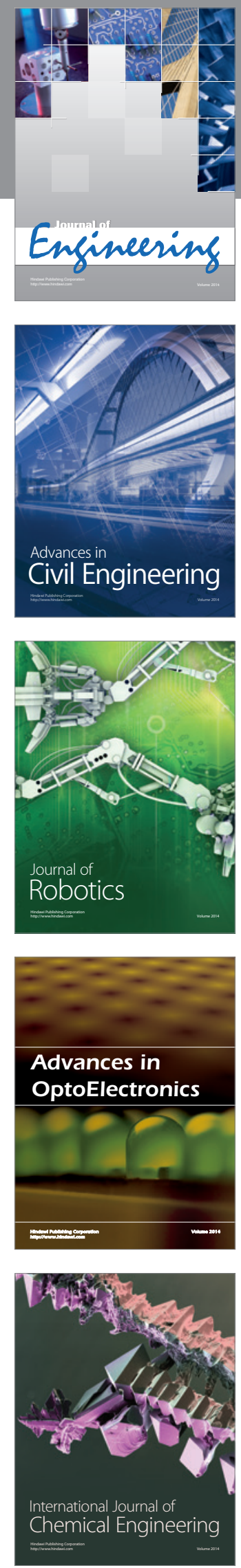

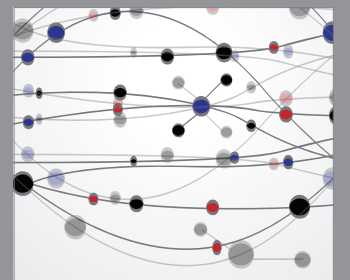

The Scientific World Journal
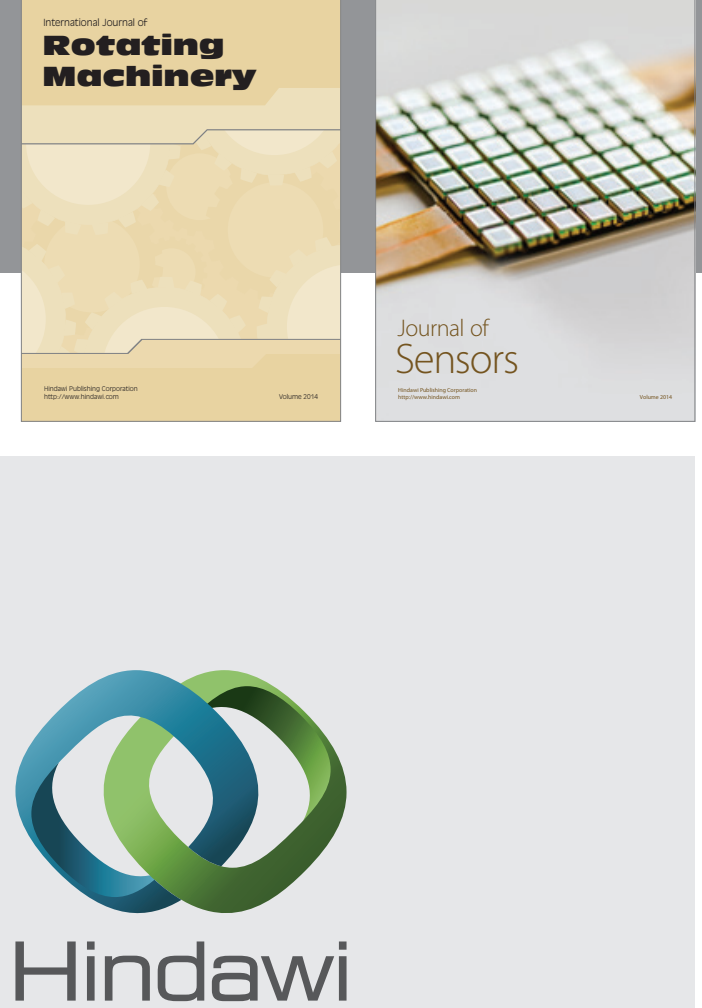

Submit your manuscripts at http://www.hindawi.com
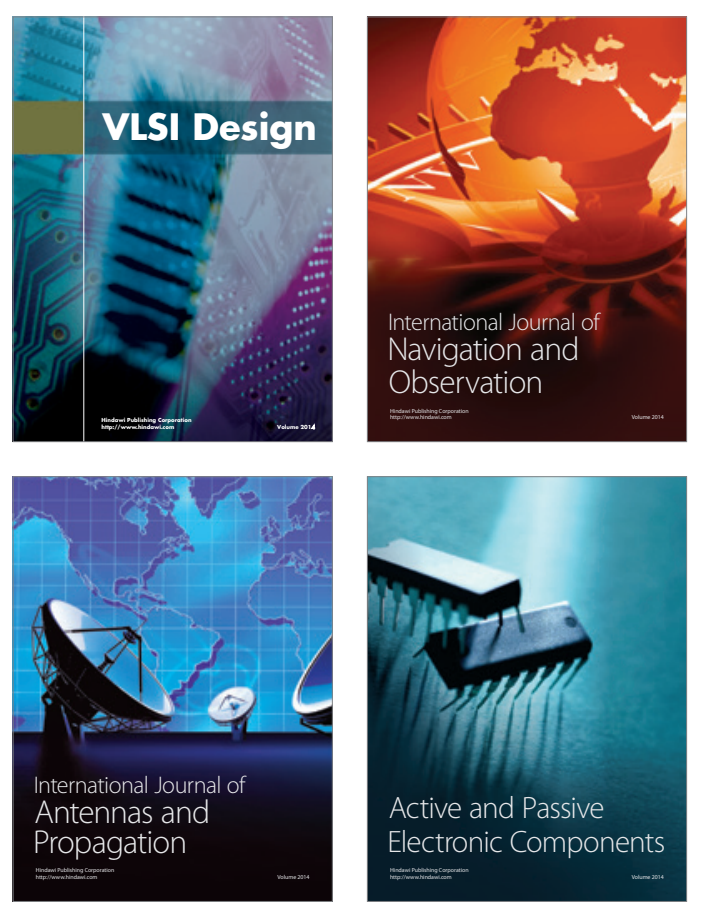
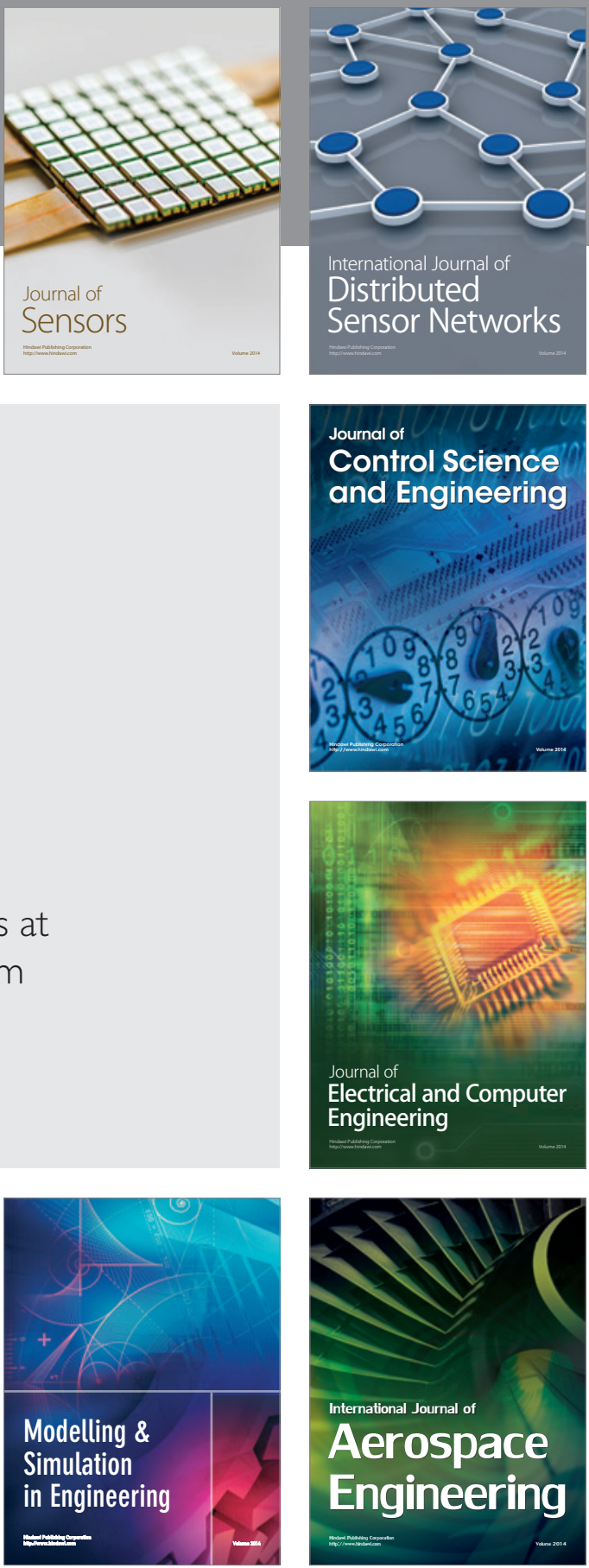

Journal of

Control Science

and Engineering
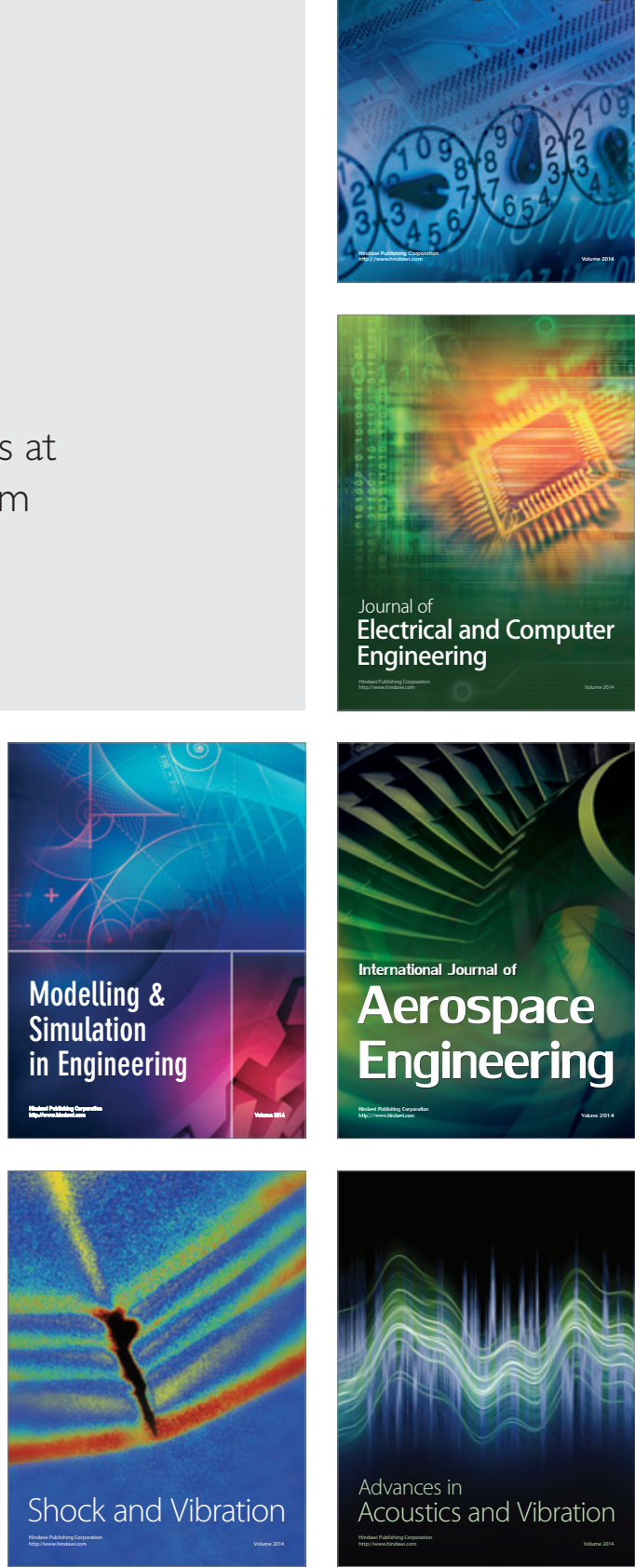